PHYSICAL REVIEW D 92, 119902(E) (2015)

\title{
Erratum: Probing Wilson loops in AdS/QCD \\ [Phys. Rev. D 91, 055001 (2015)]
}

Yizhuang Liu and Ismail Zahed

(Received 18 November 2015; published 1 December 2015)

DOI: $10.1103 /$ PhysRevD.92.119902

PACS numbers: 11.25.Tq, 13.60.Hb, 12.38.Lg, 12.38.Gc, 99.10.Cd

Equations (36) and (37) should read respectively

$$
V(L) \approx-4 k^{4} a^{4} \frac{\lambda}{N_{c}^{2}} \sum_{n=0}^{\infty}(n+1) \frac{1}{2 \pi L} e^{-k m_{X_{n}} L}
$$

and

$$
V(L) \approx-\frac{\lambda}{N_{c}^{2}} \frac{m_{\rho}^{4} a^{4}}{8 \pi L} e^{-\frac{m_{\rho} m_{X_{0}} L}{2}}
$$

with the leading asymptotic mass $m_{X 0}=\sqrt{9 / 2}$. The asymptotic behavior is consistent with the one recently reported in [1] for the Yang-Mills case. This correction was independently observed in [1].

[1] M. Giordano and E. Meggiolaro, Remarks on the static dipole-dipole potential at large distances, Phys. Rev. D 92 , 096007 (2015). 